Research Article

\title{
The Lagrangian and Hamiltonian for the Two-Dimensional Mathews-Lakshmanan Oscillator
}

\author{
Wang Guangbao $\mathbb{D}^{1}$ and Ding Guangtao ${ }^{2}$ \\ ${ }^{1}$ School of Media and Design, Chuzhou Polytechnic, No. 2188 Fengledadao, Chuzhou, Anhui 239000, China \\ ${ }^{2}$ School of Physics and Electronic Information, Anhui Normal University, Wuhu, Anhui 241000, China \\ Correspondence should be addressed to Wang Guangbao; wgb0550@163.com
}

Received 7 May 2020; Revised 27 June 2020; Accepted 14 July 2020; Published 1 August 2020

Academic Editor: Marin Marin

Copyright (c) 2020 Wang Guangbao and Ding Guangtao. This is an open access article distributed under the Creative Commons Attribution License, which permits unrestricted use, distribution, and reproduction in any medium, provided the original work is properly cited.

\begin{abstract}
The purpose of this paper is to illustrate the theory and methods of analytical mechanics that can be effectively applied to the research of some nonlinear nonconservative systems through the case study of two-dimensionally coupled MathewsLakshmanan oscillator (abbreviated as M-L oscillator). (1) According to the inverse problem method of Lagrangian mechanics, the Lagrangian and Hamiltonian function in the form of rectangular coordinates of the two-dimensional M-L oscillator is directly constructed from an integral of the two-dimensional M-L oscillators. (2) The Lagrange and Hamiltonian function in the form of polar coordinate was rewritten by using coordinate transformation. (3) By introducing the vector form variables, the two-dimensional M-L oscillator motion differential equation, the first integral, and the Lagrange function are written. Therefore, the two-dimensional M-L oscillator is directly extended to the three-dimensional case, and it is proved that the threedimensional M-L oscillator can be reduced to the two-dimensional case. (4) The two direct integration methods were provided to solve the two-dimensional M-L oscillator by using polar coordinate Lagrangian and pointed out that the one-dimensional M$\mathrm{L}$ oscillator is a special case of the two-dimensional M-L oscillator.
\end{abstract}

\section{Introduction}

Nonlinear systems are not only the traditional research fields of mechanical physics and engineering science but also the research fields of other natural science and many social science $[1,2]$. Since the middle of the last century, many researchers have been conducting research about it; they use nonlinear differential equations to simulate nonlinear dynamic system and use a variety of methods such as theory of inverse problem of variational method and conservative quantity theory to solve these equations [3-8]. As a result, the analytical mechanics theory and method in solving nonlinear differential equation have been widely used, and significant research results are achieved [9-20].

Some theoretical studies of nonlinear dynamic systems require the derivation of Lagrange functions and Hamiltonian functions corresponding to these equations and even require accurate analytical solutions. For example, in 1974, Mathews and Lakshmanan obtained a M-L oscillator subsystem by deformation of the linear conservative oscillator subsystem, which is a nonlinear nonconservative oscillator subsystem, but has a strictly controlled periodic solution $[21,22]$. Li and Ding constructed the Lagrange function of $L=\dot{x}^{2} / 2\left(1+\lambda x^{2}\right)+\omega_{0}^{2} / 2 \lambda\left(1+\lambda x^{2}\right)$ in four ways [23].

This article will systematically use analytical mechanics theory and methods to study two-dimensional M-L oscillators. First, after obtaining two integrals with clear physical meanings of the oscillator equation, a two-dimensional M-L oscillator Lagrangian function and Hamiltonian function are constructed directly from an integral according to the theory and method of Lagrangian mechanics inverse problems. And the Lagrange function and Hamiltonian function in the form of plane polar coordinates are derived by using variable transformation [24-26]. Second, the vector of position is 
introduced from the rectangular coordinates of $x$ and $y$, and the two-dimensional M-L oscillator equation and the derived grange function were written in vector form and vector variable form, respectively, thereby generalizing the twodimensional M-L oscillator to three-dimensional happening. Finally, several issues were discussed, such as the use of Lagrange functions in polar form, two methods are used to directly integrate the two-dimensional oscillator, and the Hamiltonian function can be used to further discuss the quantization of the oscillator. In this paper, by studying the analytical mechanics of the two-dimensional M-L oscillator, it is demonstrated that the methodology of analytical mechanics is of unique value in the study of some nonlinear nonconservative systems. Of which the construction of nonlinear nonconservative systems of Lagrange function and Hamiltonian function is important links.

\section{Construction of the Lagrange Function of the Two-Dimensional M-L Oscillator}

2.1. Integral of the Two-Dimensional M-L Oscillator. In 1974, Mathews and Lakshmanan introduced a one-dimensional M-L vibrator:

$$
\ddot{x}-\frac{\lambda \dot{x}^{2}}{1+\lambda x^{2}} x+\frac{\alpha^{2}}{1+\lambda x^{2}}=0 .
$$

The one-dimensional M-L oscillator (1) is a nonlinear nonconservative system, but it has a strictly controlled periodic solution $[21,22]$. In literature [23], its Lagrange function is constructed in four ways: $L=\dot{x}^{2} / 2\left(1+\lambda x^{2}\right)+\omega_{0}^{2} / 2 \lambda\left(1+\lambda x^{2}\right)$, extended to two-dimensional M-L oscillator:

$$
\begin{aligned}
& \ddot{x}=\frac{\lambda\left[\dot{x}^{2}+\dot{y}^{2}+\lambda(x \dot{y}-y \dot{x})^{2}\right] x-\alpha^{2} x}{1+\lambda\left(x^{2}+y^{2}\right)}, \\
& \ddot{y}=\frac{\lambda\left[\dot{x}^{2}+\dot{y}^{2}+\lambda(x \dot{y}-y \dot{x})^{2}\right] y-\alpha^{2} y}{1+\lambda\left(x^{2}+y^{2}\right)} .
\end{aligned}
$$

Equation (2) is completely integrable [24, 26], and the following two integrals can be derived directly from the calculation:

$$
\begin{gathered}
J=x \dot{y}-y \dot{x}, \\
E=\frac{1}{2} \frac{\left[\dot{x}^{2}+\dot{y}^{2}+\lambda(x \dot{y}-y \dot{x})^{2}\right]}{1+\lambda\left(x^{2}+y^{2}\right)}+\frac{1}{2} \frac{\alpha^{2}\left(x^{2}+y^{2}\right)}{1+\lambda\left(x^{2}+y^{2}\right)} .
\end{gathered}
$$

Integral $J$ indicates the conservation of angular momentum, and Equation (4) is the integral of energy type. If Equation (2) is interpreted as a nonlinear and nonconservative coupled vibration of a unit mass point, the former item of integral $E$ corresponded to the kinetic energy of the mass point and the latter one corresponded to the potential energy. Integral $E$ can be rewritten as

$$
E=\frac{1}{2}\left[\dot{x}^{2}+\dot{y}^{2}-\frac{\lambda(x \dot{x}+y \dot{y})^{2}}{1+\lambda\left(x^{2}+y^{2}\right)}\right]+\frac{1}{2} \frac{\alpha^{2}\left(x^{2}+y^{2}\right)}{1+\lambda\left(x^{2}+y^{2}\right)} .
$$

2.2. A Method to Construct Lagrange Equation Directly from the First Integral. According to the Lagrange mechanics, the differential equation of the system can be derived if the Lagrange equation of the system is known. The inverse problem of the Lagrange mechanics is the differential equation of the system that is known, and it is used to test whether this equation can be written into the form of the Lagrange equation and how to write it in such way $[13,14]$. Apparently, the key of the inverse problem is whether the corresponding Lagrange equation can be constructed. Among the given methods of constructing Lagrange equations, many methods are related to the first integral of the equation, and one of the direct methods is as follows [15].

If we set the differential equation of the system motion as

$$
\begin{gathered}
\ddot{q}_{\alpha}=Q_{\alpha}(t, q, \dot{q})(\alpha=1,2, \cdots, s), \\
I=I(t, q, \dot{q}),
\end{gathered}
$$

the integral satisfies the condition of $\operatorname{det}\left(\partial^{2} I / \partial \dot{q}_{\alpha} \partial \dot{q}_{\beta}\right) \neq 0$. The Lagrange equation can be expressed in the following form:

$$
L=A(t, q) I(t, q, \dot{q})+B_{\alpha}(t, q) \dot{q}_{\alpha}+B_{0}(t, q) .
$$

The undetermined factors $A, B_{a}$, and $B_{0}$ should satisfy the following equations:

$$
\begin{gathered}
\left(\frac{\partial A}{\partial t}+\frac{\partial A}{\partial q_{\beta}} \dot{q}_{\beta}\right) \frac{\partial I}{\partial \dot{q}_{\alpha}}-\frac{\partial A}{\partial q_{\alpha}} I-2 A \frac{\partial I}{\partial q_{\alpha}}-A \frac{\partial I}{\partial \dot{q}_{\beta}} \frac{\partial Q_{\beta}}{\partial \dot{q}_{\alpha}} \\
+\frac{\partial B_{\alpha}}{\partial t}+\left(\frac{\partial B_{\alpha}}{\partial \dot{q}_{\beta}}-\frac{\partial B_{\beta}}{\partial q_{\alpha}}\right) \dot{q}_{\beta}-\frac{\partial B_{0}}{\partial q_{\alpha}}=0 \quad(\alpha=1,2, \cdots, s) .
\end{gathered}
$$

2.3. Lagrange Equation of the Two-Dimensional $M-L$ Oscillator. The Lagrange function of the two-dimensional M-L oscillator (2) is constructed by using the above method. $x$ and $y$ can be set as $q_{1}$ and $q_{2}$; then, the corresponding generalized force gained from Equation (2) is as follows:

$$
\begin{aligned}
& \ddot{x}=Q_{1}=Q_{x}=\frac{\lambda\left[\dot{x}^{2}+\dot{y}^{2}+\lambda(x \dot{y}-y \dot{x})^{2}\right] x-\alpha^{2} x}{1+\lambda\left(x^{2}+y^{2}\right)}, \\
& \ddot{y}=Q_{2}=Q_{y}=\frac{\lambda\left[\dot{x}^{2}+\dot{y}^{2}+\lambda(x \dot{y}-y \dot{x})^{2}\right] y-\alpha^{2} y}{1+\lambda\left(x^{2}+y^{2}\right)} .
\end{aligned}
$$

Select the energy integral $E$ in Equation (4) as the motion integral I in Equation (7) and put it into Equation (8); the following solutions can therefore be obtained by direct calculation: 


$$
\begin{gathered}
A=1, \\
B_{1}=B_{2}=0, \\
B_{0}=\frac{\alpha^{2}\left(x^{2}+y^{2}\right)}{1+\lambda\left(x^{2}+y^{2}\right)} .
\end{gathered}
$$

Substituting this into Equation (7), a Lagrange equation of the two-dimensional M-L oscillator can be obtained:

$$
L=\frac{1}{2} \frac{\left[\dot{x}^{2}+\dot{y}^{2}+\lambda(x \dot{y}-y \dot{x})^{2}\right]}{1+\lambda\left(x^{2}+y^{2}\right)}-\frac{1}{2} \frac{\alpha^{2}\left(x^{2}+y^{2}\right)}{1+\lambda\left(x^{2}+y^{2}\right)},
$$

which can be rewritten into

$$
L=\frac{1}{2}\left[\dot{x}^{2}+\dot{y}^{2}-\frac{\left.\lambda(x \dot{x}-y \dot{y})^{2}\right]}{1+\lambda\left(x^{2}+y^{2}\right)}\right]-\frac{1}{2} \frac{\alpha^{2}\left(x^{2}+y^{2}\right)}{1+\lambda\left(x^{2}+y^{2}\right)} .
$$

When the plane polar coordinates of $r$ and $\theta$ are introduced, the transformed equation is

$$
\begin{aligned}
& x=r \cos \theta, \\
& y=r \sin \theta .
\end{aligned}
$$

In the form of plane polar coordinates, kinematic integrals are expressed as

$$
\begin{gathered}
J=r^{2} \dot{\theta}, \\
E=\frac{1}{2} \frac{\dot{r}^{2}}{1+\lambda r^{2}}+\frac{1}{2} r^{2} \dot{\theta}^{2}+\frac{1}{2} \frac{\alpha^{2} r^{2}}{1+\lambda r^{2}} \\
E=\frac{1}{2}\left(\dot{r}^{2}+r^{2} \dot{\theta}^{2}\right)-\frac{1}{2} \frac{\lambda r^{2} \dot{r}^{2}}{1+\lambda r^{2}}+\frac{1}{2} \frac{\alpha^{2} r^{2}}{1+\lambda r^{2}} .
\end{gathered}
$$

In Equations (11) and (12), the Lagrange equations are written as

$$
\begin{array}{r}
L=\frac{1}{2} \frac{\dot{r}^{2}}{1+\lambda r^{2}}+\frac{1}{2} r^{2} \dot{\theta}^{2}-\frac{1}{2} \frac{\alpha^{2} r^{2}}{1+\lambda r^{2}}, \\
L=\frac{1}{2}\left(\dot{r}^{2}+r^{2} \dot{\theta}^{2}\right)-\frac{1}{2} \frac{\lambda r^{2} \dot{\mathrm{r}}^{2}}{1+\lambda r^{2}}-\frac{1}{2} \frac{\alpha^{2} r^{2}}{1+\lambda r^{2}} .
\end{array}
$$

\section{The Hamiltonian Equation of the Three- Dimensional M-L Oscillator}

According to the Lagrange 3quation (11), the respective conjugated canonical momentums derived from the coordinates $x$ and $y$ are

$$
\begin{aligned}
& p_{x}=\frac{\partial L}{\partial \dot{x}}=\frac{\left(1+\lambda y^{2}\right) \dot{x}-\lambda x y \dot{y}}{1+\lambda\left(x^{2}+y^{2}\right)}, \\
& p_{y}=\frac{\partial L}{\partial \dot{y}}=\frac{\left(1+\lambda x^{2}\right) \dot{y}-\lambda x y \dot{x}}{1+\lambda\left(x^{2}+y^{2}\right)} .
\end{aligned}
$$

The inverse solution are

$$
\begin{aligned}
& \dot{x}=\left(1+\lambda x^{2}\right) p_{x}+\lambda x y p_{y}, \\
& \dot{y}=\left(1+\lambda y^{2}\right) p_{y}+\lambda x y p_{x} .
\end{aligned}
$$

The Hamiltonian equation of the two-dimensional M-L oscillator is obtained by means of Legendre transformation:

$$
\begin{aligned}
H & =\frac{1}{2}\left\{\left[1+\lambda\left(x^{2}+y^{2}\right)\right]\left(p_{x}^{2}+p_{y}^{2}\right)-\left(x p_{y}+y p_{x}\right)^{2}\right\} \\
& +\frac{1}{2} \frac{\alpha^{2}\left(x^{2}+y^{2}\right)}{1+\lambda\left(x^{2}+y^{2}\right)}
\end{aligned}
$$

or rewritten as

$$
H=\frac{1}{2}\left[p_{x}^{2}+p_{y}{ }^{2}+\lambda\left(x p_{x}+y p_{y}\right)^{2}\right]+\frac{1}{2} \frac{\alpha^{2}\left(x^{2}+y^{2}\right)}{1+\lambda\left(x^{2}+y^{2}\right)}
$$

Similarly, the generalized momentum and Hamilton equation corresponding to plane polar coordinates can be derived as

$$
\begin{gathered}
p_{r}=\frac{\dot{r}}{1+\lambda r^{2}} \\
p_{\theta}=r^{2} \dot{\theta} \\
H=\frac{1}{2}\left[\left(1+\lambda r^{2}\right) p_{r}{ }^{2}+\frac{1}{r^{2}} p_{\theta}{ }^{2}\right]+\frac{1}{2} \frac{\alpha^{2} r^{2}}{1+\lambda r^{2}} .
\end{gathered}
$$

\section{The Three-Dimensional Extension of the M- L Oscillator}

The vector of position is introduced from the rectangular coordinates of $x$ and $y$, the two-dimensional M-L oscillator equation and the derived grange function were written in vector form and vector variable form, respectively. From this, the two-dimensional vector is extended to three-dimensional vector, so that the two-dimensional M-L oscillator is extended to three-dimensional.

The position vector is hence introduced as

$$
\vec{r}=x \vec{i}+y \vec{j}
$$

The differential equation (2) can be written in vector form as

$$
\ddot{\vec{r}}=\frac{\lambda\left[\dot{\vec{r}}^{2}+\lambda(\vec{r} \times \dot{\vec{r}})^{2}\right]-\alpha^{2}}{1+\lambda r^{2}} \vec{r}
$$

or as 


$$
\ddot{\vec{r}}=\frac{\lambda\left[\left(1+\lambda r^{2}\right)^{\dot{r}^{2}}-\lambda(\vec{r} \cdot \dot{\vec{r}})^{2}\right]-\alpha^{2}}{1+\lambda r^{2}} \vec{r} .
$$

Correspondingly, the first integrals Equations (3), (4), and (5) are written, respectively, as

$$
\begin{gathered}
\vec{J}=\vec{r} \times \dot{\vec{r}}, \\
E=\frac{1}{2} \frac{\left[\dot{\vec{r}}^{2}+\lambda(\vec{r} \times \dot{\vec{r}})^{2}\right]}{1+\lambda r^{2}}+\frac{1}{2} \frac{\alpha^{2} r^{2}}{1+\lambda r^{2}}, \\
E=\frac{1}{2}\left[\dot{\vec{r}}^{2}-\frac{\left.\lambda(\vec{r} \cdot \dot{\vec{r}})^{2}\right]}{1+\lambda r^{2}}\right]+\frac{1}{2} \frac{\alpha^{2} r^{2}}{1+\lambda r^{2}} .
\end{gathered}
$$

In the form of vector variable, the Lagrange equation (11) is written as

$$
\begin{aligned}
& L=\frac{1}{2} \frac{\left[\dot{\vec{r}}^{2}+\lambda(\vec{r} \times \dot{\vec{r}})^{2}\right]}{1+\lambda r^{2}}-\frac{1}{2} \frac{\alpha^{2} r^{2}}{1+\lambda r^{2}}, \\
& L=\frac{1}{2}\left[\dot{\vec{r}}^{2}-\frac{\left.\lambda(\vec{r} \cdot \dot{\vec{r}})^{2}\right]}{1+\lambda r^{2}}\right]-\frac{1}{2} \frac{\alpha^{2} r^{2}}{1+\lambda r^{2}} .
\end{aligned}
$$

If the M-L oscillator is extended to the three-dimensional case, the two-dimensional plane potential vector of Equation (24) can be directly extended to the three-dimensional one.

$$
\vec{r}=x \vec{i}+y \vec{j}+z \vec{k}
$$

Therefore, Equations (25), (26), (27), (28), (29), and (30) are directly extended to three-dimensional cases without being rewritten. However, there will be changes when they are written in rectangular coordinate forms. For example, the component forms of Equation (25) will be written in rectangular coordinate forms:

$$
\begin{aligned}
& \ddot{x}=\frac{\lambda\left\{\dot{x}^{2}+\dot{y}^{2}+\dot{z}^{2}+\lambda\left[(x \dot{y}-y \dot{x})^{2}+(z \dot{x}-x \dot{z})^{2}+(y \dot{z}-z \dot{y})^{2}\right]\right\}-\alpha^{2}}{1+\lambda\left(x^{2}+y^{2}+z^{2}\right)} x, \\
& \ddot{y}=\frac{\lambda\left\{\dot{x}^{2}+\dot{y}^{2}+\dot{z}^{2}+\lambda\left[(x \dot{y}-y \dot{x})^{2}+(z \dot{x}-x \dot{z})^{2}+(y \dot{z}-z \dot{y})^{2}\right]\right\}-\alpha^{2}}{1+\lambda\left(x^{2}+y^{2}+z^{2}\right)} y, \\
& \ddot{z}=\frac{\lambda\left\{\dot{x}^{2}+\dot{y}^{2}+\dot{z}^{2}+\lambda\left[(x \dot{y}-y \dot{x})^{2}+(z \dot{x}-x \dot{z})^{2}+(y \dot{z}-z \dot{y})^{2}\right]\right\}-\alpha^{2}}{1+\lambda\left(x^{2}+y^{2}+z^{2}\right)} z .
\end{aligned}
$$

Correspondingly, there are three component conservation formulas for the angular momentum integral (27), and the energy integrals (28) and (29) should also be rewritten.
Hence, the Lagrange equation (30) is written as

$$
\begin{aligned}
L= & \frac{\left\{\dot{x}^{2}+\dot{y}^{2}+\dot{z}^{2}+\lambda\left[(x \dot{y}-y \dot{x})^{2}+(z \dot{x}-x \dot{z})^{2}+(y \dot{z}-z \dot{y})^{2}\right]\right\}}{1+\lambda\left(x^{2}+y^{2}+z^{2}\right)} \\
& -\frac{1}{2} \frac{\alpha^{2}\left(x^{2}+y^{2}+z^{2}\right)}{1+\lambda\left(x^{2}+y^{2}+z^{2}\right)} .
\end{aligned}
$$

It should be noted that the extension of the threedimensional case of the M-L oscillator has no special significance. According to the previous discussion, the expression (29) of the angular momentum conservation in vector form has gone beyond the two-dimensional space and $\vec{J}$ is perpendicular to the plane of motion. After introducing of the threedimensional position vector, the angular momentum $\vec{J}$ is still conserved and its direction remains unchanged in space. In the following formula

$$
\vec{r} \cdot \vec{J}=\vec{r} \cdot(\vec{r} \times \dot{\vec{r}}) \equiv 0
$$

it shows that the three-dimensional M-L oscillator moves in the plane $\vec{J}$ perpendicular to the constant vector, passing through the origin. If the $z$-axis is parallel to $\vec{J}$, the oscillator must move in the $x y$-plane, that is,

$$
z \equiv 0
$$

In such a coordinate system, Equation (33) degenerates to Equation (2), and Lagrange equation (34) is reduced to Equation (11). In other words, the extended three-dimensional M-L oscillator is reduced to a two-dimensional one. The following section will point out that the one-dimensional M-L oscillator (1) is a special case of the two-dimensional one.

\section{Discussion}

After Obtaining the Lagrange function in polar form, the two-dimensional M-L oscillator can be solved directly by two methods:

Approach 1. The Lagrangian function of the radial motion of the oscillator can be derived by using Equations (14) and (17).

$$
L_{r}=\frac{1}{2} \frac{\dot{r}^{2}}{1+\lambda r^{2}}+\frac{1}{2} \frac{J^{2}}{r^{2}}+\frac{1}{2} \frac{\alpha^{2} r^{2}}{1+\lambda r^{2}} \text {. }
$$

Now the motion of the two-dimensional M-L oscillator has been reduced to the problem of one-dimensional radial motion, which can be directly integrated.

Approach 2. As can be seen from Equation (28), if $J=0$, the two-dimensional M-L oscillator will degenerate to the M-L oscillator represented by Equation (1); if $J \neq 0$, the differential quotient of time $t$ can be transformed into that of the space coordinate by using the integral of Equation (14): 


$$
\frac{d}{d t}=\dot{\theta} \frac{d}{d \theta}=\frac{J}{r^{2}} \frac{d}{d \theta}
$$

An orbital differential equation of a two-dimensional M-L oscillator can be derived by substituting the energytype integral (15).

$$
\frac{J^{2}}{2}\left[\frac{1}{r^{4}\left(1+\lambda r^{2}\right)}\left(\frac{d r}{d \theta}\right)^{2}+\frac{1}{r^{2}}\right]+\frac{1}{2} \frac{\alpha^{2} r^{2}}{1+\lambda r^{2}}=E
$$

By integrating this equation, the orbit equation of the oscillator $(r=r(\theta))$ can be obtained, and then, $\theta=\theta(t)$ is hence obtained when plugging in integral (14) of the angular momentum.

In the study of modern physics, for example, it may involve the quantization of some nonconservative nonlinear systems when dealing with some mesoscopic physical systems. At this time, it is often necessary to derive the Hamiltonian function of the system. The Hamiltonian function of the twodimensional M-L oscillator derived in this paper can be directly solved by Hamiltonian mechanics methods, such as HamiltonJacobi theory; on the other hand, it also lays the foundation for further discussion of the quantization of this system.

\section{Conclusions}

There are many ways to study nonlinear and nonconservative systems, but analytical mechanics theories and methods have an important value in such research. In some studies, e.g., discussing the quantization problem, it is necessary to first mechanize the system analysis; that is to say, the Lagrange and Hamiltonian functions of the system need to be derived first. In this paper, according to the Lagrange mechanics inverse problem theory and method, the Lagrange function and Hamiltonian function of the oscillator can be constructed directly from the energy form integral of the two-dimensional M-L oscillator, which can be used to realize the analytical mechanics of the nonlinear and nonconservative system. The two-dimensional M-L vibrator is solved by using traditional analytical mechanics methods such as coordinate transformation and motion integration reduction order separation variables. At the same time, the relationship between the two-dimensional M-L oscillator and the onedimensional and three-dimensional M-L oscillator is discussed. By studying the analytical mechanics of the twodimensional M-L oscillator, it is shown that the methodology of analytical mechanics is of vital value in the study of some nonlinear nonconservative systems. The key lies in the analysis and mechanization of nonlinear differential equations of motion, meaning that the Lagrange function and Hamilton function of nonlinear equations can be derived by using analytical mechanics inverse problem methodology.

\section{Data Availability}

No data were used to support this study of "The Lagrangian and Hamiltonian for the Two-dimensional MathewsLakshmanan Oscillator."

\section{Conflicts of Interest}

The authors declare no conflict of interest.

\section{Acknowledgments}

The authors gratefully acknowledge the financial support by Natural Science Research Project of Anhui Education Department (PX-271191861) and School Enterprise Cooperation Practical Education Base of Anhui Education Department (2017sjjd050).

\section{References}

[1] B. Z. Liu, Nonlinear Dynamics, Higher Education Press, Beijing, China, 2004.

[2] Y. Z. Liu and L. Q. Chen, Nonlinear Vibration, Higher Education Press, Beijing, China, 2001.

[3] M. Marin, E. M. Craciun, and N. Pop, "Considerations on mixed initial-boundary value problems for micropolar porous bodies," Dynamic Systems and Applications, vol. 25, no. 1-2, pp. 175-196, 2016.

[4] C. Itu, A. Ochsner, S. Vlase, and M. I. Marin, "Improved rigidity of composite circular plates through radial ribs," Proceedings of the Institution of Mechanical Engineers, Part L: Journal of Materials: Design and Applications, vol. 233, no. 8, pp. 1585-1593, 2018.

[5] M. M. Bhatti, R. Ellahi, A. Zeeshan, M. Marin, and N. Ijaz, "Numerical study of heat transfer and Hall current impact on peristaltic propulsion of particle-fluid suspension with compliant wall properties," Modern Physics Letters B, vol. 33, no. 35, article 1950439, 2019.

[6] M. Marin, R. Ellahi, S. Vlase, and M. M. Bhatti, "On the decay of exponential type for the solutions in a dipolar elastic body," Journal of Taibah University for Science, vol. 14, no. 1, pp. 534540, 2020.

[7] M. Marin, E. M. Craciun, and N. Pop, "Some results in GreenLindsay thermoelasticity of bodies with dipolar structure," Mathematics, vol. 8, no. 4, p. 497, 2020.

[8] M. Marin, A. Öchsner, and E. M. Craciun, "A generalization of the Gurtin's variational principle in thermoelasticity without energy dissipation of dipolar bodies," Continuum Mechanics and Thermodynamics, pp. 1-10, 2020.

[9] R. M. Santilli, Foundations of Theoretical Mechanics I, Springer-Verlag, New York, NY, USA, 1978.

[10] F. X. Mei, Symmetries and Conserved Quantities of Constrained Mechanical Systems, Beijing Institute of Technology Press, Beijing, China, 2004.

[11] Z. E. Musielak, D. Roy, and L. D. Swift, "Method to derive Lagrangian and Hamiltonian for a nonlinear dynamical system with variable coefficients," Chaos, Solitons \& Fractals, vol. 38, no. 3, pp. 894-902, 2008.

[12] S. K. Luo and Y. F. Zhang, Advances in the Study of Dynamics of Constrained Systems, Science Press, Beijing, China, 2008.

[13] D. J. Saunders, "Thirty years of the inverse problem in the calculus of variations," Reports on Mathematical Physics, vol. 66, no. 1, pp. 43-53, 2010.

[14] G. T. Ding, "A direct approach to the construction of Lagrangians from the motion equation," Journal of Dynamics and Control, vol. 8, pp. 305-310, 2010. 
[15] G. T. Ding, "A new approach to construction of the Lagrangians and Hamiltonians for one-dimensional dissipative systems with variable coefficients," Acta Physica Sinica, vol. 60, article 444503, 2011.

[16] G. T. Ding, "A direct approach to construction of the Lagrangian from the first integral," Journal of Dynamics and Control, vol. 9, pp. 102-106, 2011.

[17] F. X. Mei and H. B. Wu, Methods of Analytical Mechanics for Solving Differential Equations, Sceince Press, Beijing, China, 2012.

[18] B. Chen, Analytical Dynamics, Beijing University Press, Beijing, China, 2nd edition, 2012.

[19] G. T. Ding, "The construction of the Lagrangians for dissipative-like systems by using the transformations of variables," Journal of Dynamics and Control, vol. 10, pp. 199201, 2012.

[20] G. T. Ding, "Two methods to derive Lagrangians for a nonlinear dynamical system with variable coefficients," Journal of Dynamics and Control, vol. 15, no. 1, pp. 10-14, 2017.

[21] P. M. Mathews and M. Lakshmanan, "On a unique nonlinear oscillator," Quarterly of Applied Mathematics, vol. 32, no. 2, pp. 215-218, 1974.

[22] C. Quesne, "Generalized nonlinear oscillators with quasiharmonic behaviour: classical solutions," Journal of Mathematical Physics, vol. 56, no. 1, article 012903, 2015.

[23] J. Y. Li and G. T. Ding, "Analytical mechanics methods for solving Mathews-Lakshmanan oscillator," Journal of Dynamics and Control, vol. 16, pp. 1-5, 2018.

[24] V. K. Chandrasekar, M. Senthilvelan, and M. Lakshmanan, "On the complete integrability and linearization of certain second-order nonlinear ordinary differential equations," Proceedings of the Royal Society A: Mathematical, Physical and Engineering Sciences, vol. 461, no. 2060, pp. 2451-2477, 2005.

[25] V. K. Chandrasekar, S. N. Pandey, M. Senthilvelan, and M. Lakshmanan, "A simple and unified approach to identify integrable nonlinear oscillators and systems," Journal of Mathematical Physics, vol. 47, no. 2, article 023508, 2006.

[26] J. F. Cariñena, M. F. Rañada, and M. Santander, "Three superintegrable two-dimensional oscillators: superintegrability, nonlinearity, and curvature," Physics of Atomic Nuclei, vol. 70, no. 3, pp. 505-512, 2007. 